\title{
Reinnervation Accuracy of the Rat Femoral Nerve by Motor and Sensory Neurons
}

\author{
Roger D. Madison, ${ }^{1,2,3}$ Simon J. Archibald, ${ }^{1}$ and Thomas M. Brushart ${ }^{4,5}$ \\ ${ }^{1}$ Division of Neurosurgery and 2 Department of Neurobiology, Duke University Medical Center, Durham, North Carolina \\ 27710, ${ }^{3}$ Research Service of the Veterans Affairs Medical Center, Durham, North Carolina 27705, ${ }^{4}$ Departments of \\ Orthopedics and Neurology, The Johns Hopkins Hospital, Baltimore, Maryland 21205, and ${ }^{5}$ The Raymond M. Curtis \\ Hand Center, Baltimore, Maryland 21218
}

Previous studies in the rat femoral nerve have shown that regenerating motor neurons preferentially reinnervate a terminal nerve branch to muscle as opposed to skin, a process that has been called preferential motor reinnervation. However, the ability of sensory afferent neurons to accurately reinnervate terminal nerve pathways has been controversial. Within the dorsal root ganglia, sensory neurons projecting to muscle are interspersed with sensory neurons projecting to skin. Thus, anatomical studies assessing the accuracy of sensory neuron regeneration have been hampered by the inability to reliably determine their original innervation status. A sensory neuron that regenerated an axon into a terminal nerve branch to muscle might represent either an appropriate return of an original sensory afferent to muscle stretch receptors or the inappropriate recruitment of a cutaneous sensory afferent that originally innervated skin. The current experiments used a labeling strategy that effectively labels motor and sensory neurons projecting to a terminal nerve branch before experimental manipulation of the parent mixed nerve. Our results confirm previous observations concerning preferential motor reinnervation for motor neurons, and show for the first time anatomical evidence of specificity during regeneration of sensory afferent projections to muscle. In addition, the accuracy of sensory afferent regeneration was highly correlated with the accuracy of motor regeneration. This suggests that these two distinct neuronal populations that project to muscle respond in parallel to specific guidance factors during the regeneration process.

Key words: PNS; axonal regeneration accuracy; preferential motor reinnervation; rat femoral nerve; pathway guidance; axon growth
Functional peripheral nerve regeneration depends on interactions among regenerating axons, non-neuronal cells, growth factors and their receptors, cell adhesion molecules, and extracellular matrix materials (for review, see Fields et al., 1989; Hall, 1989; Fawcett and Keynes, 1990; Madison et al., 1992; Raivich and Kreutzberg, 1993). Given the complexity of these interactions, it is not surprising that the clinical outcome of nerve repair often is poor (Cooney, 1991). Regenerating motor axons often are misrouted to cutaneous targets, whereas sensory axons formerly innervating skin often are misrouted to muscle (Brushart, 1994). Nevertheless, under favorable conditions, axonal regeneration in the mammalian peripheral nervous system can occur with remarkable accuracy and result in significant return of function. This paper explores the accuracy with which regenerating motor axons and muscle sensory afferents reinnervate their original terminal nerve branch. Although this study does not speak to actual distal receptor reinnervation, it is important to emphasize that if accurate choices are not made at the terminal nerve branch level, progressively finer discriminations might be rendered impossible by the lack of appropriate end organ choices available at the termination of the tributary nerve.

The rat femoral nerve has several anatomical characteristics

Received May 9, 1996; revised June 14, 1996; accepted June 24, 1996.

This study was supported by National Institutes of Health Grant NS22404-11 (R.M.), the Merit Review Program of the U.S. Department of Veterans Affairs (R.M.), and The Raymond M. Curtis Research Fund (T.M.B.). We thank Mr. Steven Meadows and Mr. Philip Kessens for excellent technical assistance.

Correspondence should be addressed to Roger D. Madison, Division of Neurosurgery, Box 2609, Duke University Medical Center, Durham, NC 27710.

Copyright $\odot 1996$ Society for Neuroscience $0270-6474 / 96 / 165698-06 \$ 05.00 / 0$ that make it an ideal model for this investigation (Weiss and Edds, 1945; Brushart, 1988). Proximally, in the retroperitoneum, axons destined for muscle and skin intermingle; lesions at this site provide random access to both cutaneous and muscle Schwann cell tubes in the distal nerve stump. Distally, these axons segregate into a terminal muscle branch to the quadriceps and a cutaneous branch to the leg; the cutaneous branch normally contains no motor axons, thus reinnervation by motor neurons represents a failure of specificity (Brushart, 1988). Additionally, the quadriceps branch $(\mathrm{QB})$ and the cutaneous branch $(\mathrm{CB})$ are well matched as competing "targets" for regenerating axons; overall, approximately equal areas of axoplasm are exposed on cross-section of the two branches.

It has been demonstrated previously that motor neurons preferentially reinnervate the $\mathrm{QB}$ and/or quadriceps muscle when given equal access to cutaneous and muscle pathways, a process called preferential motor reinnervation (PMR) (Brushart, 1988). PMR was observed even when the repair was intentionally misaligned or a gap was imposed between proximal and distal stumps. Simultaneous application of tracers to reinnervated QB and CB (simultaneous double labeling) revealed that axon collaterals of many motor neurons initially entered both distal pathways (Brushart, 1990a). Specificity then was generated by pruning collaterals from cutaneous pathways while maintaining those in muscle pathways.

However, the simultaneous labeling paradigm cannot assess the accuracy with which muscle afferents return to muscle as opposed to skin, because their neurons intermingle within the dorsal root ganglia (DRG) and cannot be separated anatomically. An addi- 


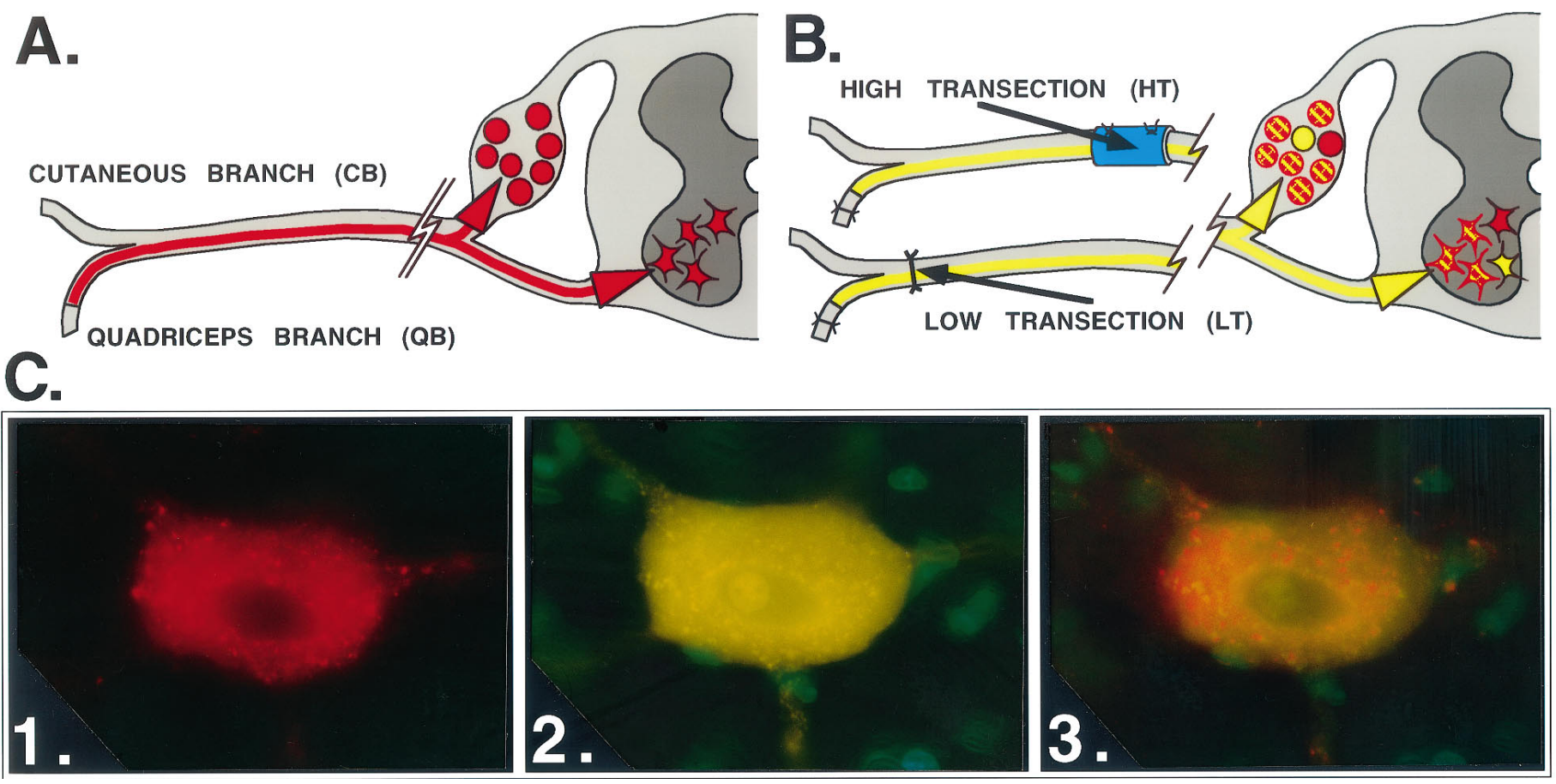

Figure 1. Femoral nerve model for determining regeneration specificity. $A$, Initial retrograde labeling with DiI of the motor and sensory neuron pools innervating the terminal branch of the femoral nerve to the QB before any experimental manipulation of the femoral nerve. $B$, Secondary retrograde labeling with FG of the motor and sensory neurons that regenerated axons into the QB 4 weeks after transection and repair of the parent femoral nerve. One of two repair procedures was used: either an entubulation repair of a high femoral nerve transection $(H T)$ or a direct suture repair of a low femoral nerve transection $(L T)$. The suture marks distal to the FG application site indicate the location of the previous exposure to DiI. $C$, Photomicrographs of a regenerated motor neuron labeled with both tracers: 1. rhodamine optics (DiI), 2. FG optics, and 3. a double exposure using successive FG and rhodamine filter sets.

tional limitation is that motor neurons that originally projected to the iliacus muscle (innervated midway between a proximal repair site and the distal bifurcation), but now are projecting to the QB, are impossible to differentiate from original quadriceps motor neurons, because they both share a single motoneuron pool.

These defects were overcome by labeling motor and sensory neurons projecting to the QB with a fluorescent dye, DiI, before experimental manipulation of the femoral nerve (Harsh et al., 1991; Madison and Archibald, 1992; Madison et al., 1992). This dye remains stable within neurons (Godement et al., 1987; Harsh et al., 1991; Madison et al., 1992). A second tracer application to the QB after recovery from a proximal femoral nerve lesion presents the question, "How many neurons (sensory or motor) that originally innervated the quadriceps muscle have returned to this terminal nerve branch?"

\section{MATERIALS AND METHODS}

Experiments were performed on $250 \mathrm{gm}$ Sprague Dawley rats under deep anesthesia with either Chloropent ( $3 \mathrm{ml} / \mathrm{kg}$ ) (Dodge Labs) or $2 \mathrm{ml} / \mathrm{kg}$ of a solution containing ketamine $(50 \mathrm{mg} / \mathrm{ml})$, xylazine $(2.6 \mathrm{mg} / \mathrm{ml})$, and acepromazine maleate $(0.5 \mathrm{mg} / \mathrm{ml})$. The QB was sharply transected $2 \mathrm{~mm}$ proximal to the quadriceps muscle, the proximal stump drawn into a 3- to 4-mm-long polyethylene tube $(1.57 \mathrm{~mm}$, inner diameter) (Clay Adams), and the base of the tube sealed around the nerve entry site using petroleum jelly as described previously (Harsh et al., 1991). The tube was filled with a 3\% suspension of DiI (Molecular Probes, Eugene, OR). After a $1 \mathrm{hr}$ exposure to DiI, the polyethylene tube with the DiI was carefully removed, the proximal nerve stump cleaned with saline to remove excess DiI, and the QB sutured with two 10-0 nylon sutures.

Two weeks later, animals in the experimental groups received transection of the proximal parent femoral nerve. The low-transection (LT) group $(n=9)$ (Fig. 1) received transection and direct suture repair of the femoral nerve $10 \mathrm{~mm}$ proximal to the terminal bifurcation of the nerve.
This level is distal to the branches to the iliacus muscle, and is at a level where the axons to the QB have coalesced within the lateral half of the nerve. The high-transection (HT) group $(n=14)$ (Fig. 1) received transection of the femoral nerve $20 \mathrm{~mm}$ proximal to the terminal bifurcation, proximal to the iliacus branch. At this very proximal level, axons destined for the QB are dispersed throughout the entire nerve (Brushart, 1988). As an additional attempt to defeat any influence of guidance caused by mechanical alignment, in the HT group the nerve was repaired with a silicone tube (0.025 inch, inner diameter) (Dow Corning, Corning, $\mathrm{NY}$ ) to impose an unstructured $0.5 \mathrm{~mm}$ gap between proximal and distal nerve stumps.

Four weeks after femoral nerve transection and repair, the QB was retransected just proximal to the site of DiI application and exposed to $5 \%$ Fluorogold (FG) using the same technique as for DiI (Fluorochrome, Englewood, CO). Seventy-two hours after FG application, animals were perfused transcardially with heparinized PBS, $\mathrm{pH} 7.2$, followed by $4 \%$ paraformaldehyde in $0.1 \mathrm{M}$ phosphate buffer, $\mathrm{pH}$ 7.2. Tissues (femoral nerve repair site, L2-L4 DRG, spinal cord) were removed and post-fixed overnight ( $20 \%$ sucrose, $4 \%$ paraformaldehyde in $0.1 \mathrm{M}$ phosphate buffer at $4^{\circ} \mathrm{C}$ ). Serial $24 \mu \mathrm{m}$ cryostat sections of the spinal cord and DRG were collected into phosphate buffer, wet mounted, and coverslipped with $40 \%$ glycerol in $0.1 \mathrm{M}$ sodium carbonate, $\mathrm{pH}$ 9.0, containing $0.04 \%$ $p$-phenylenediamine (Dodd et al., 1984). Tissues were kept wet throughout the dissection and mounting process, because air drying the sections diminishes the intensity of DiI. Sections were examined on a Zeiss Axiophot microscope using the recommended filters for rhodamine (DiI) and low UV (FG), as described previously (Harsh et al., 1991). Singleand double-labeled neurons were quantified by an observer who was unaware of the group identity of the tissue, and all counts were corrected according to Konigsmark (1970).

For these experiments to be meaningful, it must be shown that both dyes label not only an equal number of neurons, but exactly the same neurons when they are applied to the same nerve trunk at widely separate times. Ten control animals received exposure to DiI as described above, and 6 weeks later the same QB was exposed to FG. These control animals 
thus survived for the same period after DiI labeling as did the experimental animals.

The size distribution of 100 double-labeled motor neurons each for one of the control animals and one of the regenerated cases was calculated to estimate the relative numbers of $\tau$ versus $\alpha$ motor neurons. Cell size was determined from measurements of the cell perimeter as described previously (Swett et al., 1986). A size of $<30 \mu$ was used to indicate $\tau$ motor neurons, and $>30 \mu$ to indicate $\alpha$ motor neurons (Swett et al., 1986).

The repair sites of four of the HT animals were processed for plastic embedding. Standard morphometric procedures were used to count the number of myelinated axons proximal and distal to the transection site (Cordeiro et al., 1989; Archibald et al., 1995). One unoperated femoral nerve was processed in a similar manner.

\section{RESULTS}

The representative quality and extent of labeling produced by DiI and FG are demonstrated in Figure 1. Quantitative analysis of such sections is straightforward, and if the microscope slides are stored at $4^{\circ} \mathrm{C}$, the label persists for several months. In addition, if the spinal cord is removed and stored in fixative overnight and then in PBS, the labeling persists for up to 1 year (R. Madison and S. Archibald, unpublished observations).

Neurons that contained both tracers originally projected to the QB and "correctly" regenerated an axon back to that branch (Fig. 1). Neurons that contained only the first tracer (DiI) originally projected to the QB but failed to return. This could result from misrouting of the regenerating axon into the terminal $\mathrm{CB}$ or iliacus branch or from failure to regenerate all together. Finally, neurons containing only the second tracer (FG) initially innervated other structures and inappropriately reinnervated the QB after femoral nerve transection.

The control animals proved that the same population of neurons could be labeled over time when DiI and FG were successively applied to the QB. This labeling protocol resulted in $>96 \%$ of original neurons being labeled with both dyes (Table 1) (see also Harsh et al., 1991). In the control studies, only labeled neurons in the L3 DRG were counted as a representative sample. In the experimental animals, the entire population of labeled L2-L4 DRG neurons was quantified.

The percent correct motor reinnervation after femoral nerve transection and repair (double label/total DiI pool $\times 100$ ) is shown in Figure 2 and Table 2. We chose to analyze the data as "percent correct" to compensate for any variation between the absolute number of neurons in the neuronal pools. This normalization highlights that the important variable is the proportion of neurons returning to their original nerve branch, rather than the absolute neuronal pool in any particular animal. Significantly more original motor neurons returned in the LT (78\%) than in the HT (59\%) group. The total DiI pool, or the original neuronal pool to the QB, was not different between the two groups, and both were similar to control values $(394 \pm 20$; mean \pm SEM; $n=10)$.

Likewise, significantly more original sensory afferent neurons projected to the QB in the LT $(76 \%)$ versus HT (49\%) group (Fig. 2, Table 3). Unlike the total DiI pools for motor neurons, there was a significant reduction in the total DiI pool of sensory neurons for the HT group compared with the LT group (660 vs 1550; $p<$ 0.001). This reduction probably reflects the death of some DRG neurons because of the more proximal transection (Arvidsson et al., 1986; Himes and Tessler, 1989). There was a trend within each transection group for more motor neurons compared with sensory afferent neurons to project back to the QB; however, this trend was not statistically significant.

A statistical analysis using a one-sample $Z$ test was carried out between the observed accuracy of the number of returning neurons compared with that expected if regeneration was random (Zar, 1984). Both repair conditions resulted in significantly more neurons (motor and sensory) projecting back to the QB than predicted by chance ( $p<0.0001, Z$ test) (see Discussion).

There also was a significant positive correlation $(p<0.001)$ between motor and sensory neurons in terms of the percent of "correct" regeneration (Fig. 3). This correlation was evident within each repair group.

\section{DISCUSSION}

The major finding of these experiments is that both motor and sensory neurons to the quadriceps muscle demonstrate a preference to reinnervate their original terminal nerve branch (QB) after parent femoral nerve transection. In the HT group, axons destined for the QB are dispersed across the entire cross-sectional area of the nerve, and a $0.5 \mathrm{~mm}$ nerve gap further prevented direct alignment of axons in the proximal and distal nerve stumps. Therefore, the degree to which distal repair (LT) provides increased specificity is an index of mechanical (axon alignment) contributions to regeneration specificity in both sensory and motor systems.

\section{Sequential double labeling (control studies)}

In control animals, $>96 \%$ of the original neuronal pools were double labeled. Although previous studies have used different tracer combinations to assess accuracy of nerve regeneration, they have been hampered by dye leakage (Fritzsch and Wilm, 1990), toxicity (Illert et al., 1982), or the need to compromise between two protocols, which results in reduced overall sensitivity (Wigston and Kennedy, 1987; Duffy et al., 1990). The use of DiI and FG circumvents these shortcomings (see also Fritzsch and Sonntag, 1991). Taken as a whole, these data indicate that sequential double labeling is a reliable tool for examining the specificity with which a neuron reinnervates its original nerve branch.

A potential confounding issue is the conditioning effect delivered to axons of the QB during the initial DiI exposure. When the entire femoral nerve is transected 2 weeks later, conditioned quadriceps motoneurons might behave differently than unconditioned iliacus and pectineus motoneurons. However, recent experiments in the femoral nerve model (Brushart, 1996) have shown that conditioning by crushing both QB and CB does not

Table 1. Control studies for double labeling

\begin{tabular}{|c|c|c|c|c|c|}
\hline Control animals (Mean \pm SEM) & $\begin{array}{l}\text { DiI pool (Total possible } \\
\text { to regenerate) }\end{array}$ & Double labeled & DiI alone & FG alone & $\begin{array}{l}\% \text { Double } \\
\text { labeled }\end{array}$ \\
\hline Motor neurons to QB $(n=10)$ & $394 \pm 20$ & $392 \pm 20$ & $2 \pm 1$ & $4 \pm 3$ & $99 \pm 1$ \\
\hline L3 DRG sensory aff. to QB $(n=6)$ & $604 \pm 80$ & $578 \pm 75$ & $14 \pm 7$ & $12 \pm 7$ & $96 \pm 2$ \\
\hline
\end{tabular}

The terminal QB was exposed to DiI, and 6 weeks later, FG. Virtually all neurons are double labeled, proving the ability to prelabel neuronal projections to the QB. 
A)

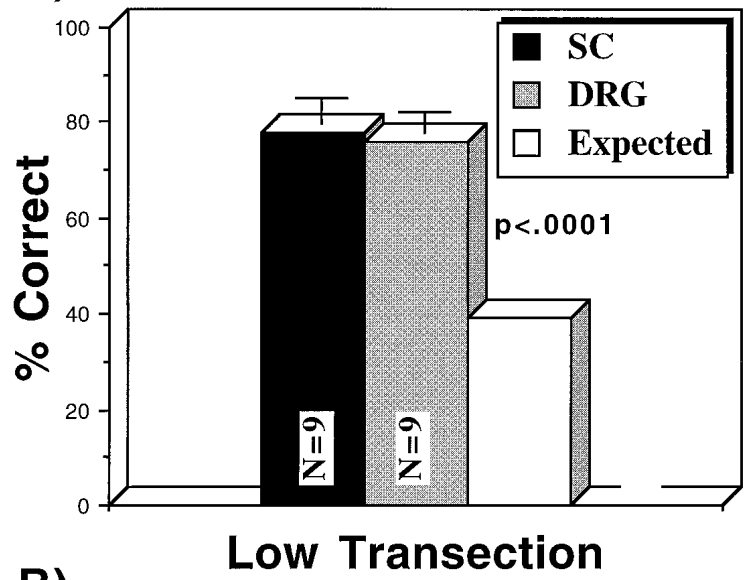

B)

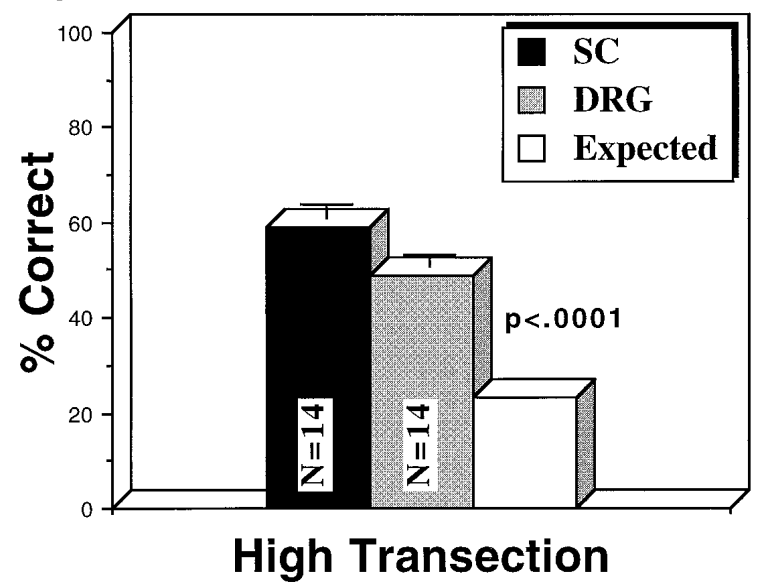

Figure 2. Percent correct regeneration of original neuronal pools to terminal QB. Neurons that contained both tracers originally projected to the terminal $\mathrm{QB}$, and "correctly" regenerated an axon back to that branch. This figure shows the "percent correct regeneration" of the original spinal cord motor $(S C)$ and sensory neurons from L2-L4 DRG to the QB after the various repair procedures. These calculations are derived by taking the number of double-labeled neurons divided by the total DiI pool $\times 100(\bar{X} \pm$ SEM) $($ see also Tables 2,3 ). There were significantly more original neurons returning in the LT group compared with the HT group for both motor and sensory neurons (ANOVA followed by Scheffe $F$ test, confirmed by Mann-Whitney $U$ test). We also can statistically compare the difference between the expected and the observed percentages by using a one-sample $Z$ test (Zar, 1984). All repair conditions resulted in significantly more neurons returning to the QB than predicted by chance $(p<0.0001, Z$ test $)$.

alter the specificity of motor axon regeneration after a later, more proximal lesion.

\section{Motor neuron regeneration to QB}

The percent correct regeneration was significantly greater for the LT compared with the HT group (78 vs 59\%; $p<0.05$ ). Motor neurons labeled with FG alone probably represent neurons that previously innervated the pectineus and iliacus muscles, but that regenerated down the QB. The influence of repair level confirms this possibility. The iliacus branch leaves the femoral nerve between high and low repair sites; iliacus axons thus would be transected in a high lesion, but not in a low lesion. A mean of 96 motoneurons inappropriately reinnervated the QB after high repair, and a mean of 29 after low repair, consistent with this mechanism. Axons serving the pectineus muscle leave the nerve just proximal to the terminal branches, thus these could be misrouted even after LT and may account for the misrouting after low repair.

Using previously published morphometric methods (Swett et al., 1986), we classified $17 \%$ of double-labeled neurons as $\tau$ motor neurons and $83 \%$ as $\alpha$ motor neurons in both control and regenerated groups (data not shown). Thus, both $\alpha$ and $\tau$ quadriceps motoneurons demonstrate a strong tendency to reinnervate the QB regardless of the level of femoral nerve transection. The slight decrease in the total motoneuron pool in the HT group compared with the LT group may represent a small percentage of cell death attributable to the very proximal femoral nerve transection.

\section{Sensory afferent regeneration to QB}

Significantly more original sensory afferent neurons returned to the QB after LT than after HT (76 vs $49 \%$; $p<0.001)$ (Fig. $2 B$, Table 3). There also was a significant reduction in the total sensory neuron DiI pool after HT as compared with LT because of sensory neuron death after the more proximal transection (660 vs $1550 ; p<0.001)$.

It is important to relate the observed regeneration accuracy to the QB with what would be expected if regeneration were simply random. To estimate such probabilities, the complexities of axonal pathway choices at the nerve transection site must be taken into account. Many studies have shown that regenerating axons exhibit a strong preference to grow along the inside portion of remaining basal lamina tubes in the distal nerve stump (Holmes and Young, 1942; Ide, 1983; Scherer and Easter, 1984). Basal lamina tubes, along with their confined Schwann cells, are known as "bands of Bungner" (Ramon y Cajal, 1928). Schwann cells originally associated with myelinated axons form this type of Schwann cell tube all the way from the transection site to the distal end organ target. Once an axon enters these continuous Schwann cell tubes at the repair site, it will be directed to the previously innervated end organ target (Brown and Hopkins, 1981; Brown and Hardman, 1987; Lee and Farel, 1988). Thus, the eventual distal destination of regenerating axons is determined by the Schwann cell tubes that they enter at the nerve transection site.

The number of myelinated axons in the QB was used to estimate the number of "hard-wired" pathways at the femoral nerve transection site that eventually enter the QB. The number of axons at the level of the transection sites and the terminal nerve branches was determined in a normal animal processed using

Table 2. Single- and double-labeled motor neurons 4 weeks after femoral nerve transection and repair

\begin{tabular}{|c|c|c|c|c|c|}
\hline $\begin{array}{l}\text { Motor neurons to } \\
\text { QB (Mean } \pm \text { SEM) }\end{array}$ & DiI pool & $\begin{array}{l}\text { Double labeled } \\
\text { (no. correct) }\end{array}$ & $\begin{array}{l}\text { DiI alone } \\
\text { (did not return) }\end{array}$ & $\begin{array}{l}\text { FG alone } \\
\text { (inappropriate } \\
\text { recruitment) }\end{array}$ & $\begin{array}{l}\% \text { Correct } \\
\text { regeneration to } \mathrm{QB}\end{array}$ \\
\hline $\mathrm{HT}(n=14)$ & $369 \pm 30$ & $218 \pm 21$ & $150 \pm 18$ & $96 \pm 12$ & $59 \pm 3$ \\
\hline $\mathrm{LT}(n=9)$ & $467 \pm 26$ & $\begin{array}{l}365 \pm 31 \\
* \text { vs HT group }\end{array}$ & $102 \pm 25$ & $\begin{array}{l}28 \pm 8 \\
* \text { vs HT group }\end{array}$ & $\begin{array}{l}78 \pm 5 \\
* \text { vs HT group }\end{array}$ \\
\hline
\end{tabular}




\begin{tabular}{|c|c|c|c|c|c|}
\hline $\begin{array}{l}\text { L2-L4 DRG } \\
\text { (sensory aff. to QB } \\
\text { Mean } \pm \text { SEM) }\end{array}$ & $\begin{array}{l}\text { DiI pool } \\
\text { (total possible to } \\
\text { regenerate) }\end{array}$ & $\begin{array}{l}\text { Double labeled } \\
\text { (no. correct) }\end{array}$ & $\begin{array}{l}\text { DiI alone } \\
\text { (did not return) }\end{array}$ & $\begin{array}{l}\text { FG alone } \\
\text { (inappropriate } \\
\text { recruitment) }\end{array}$ & $\begin{array}{l}\% \text { Correct } \\
\text { regeneration to QB }\end{array}$ \\
\hline $\mathrm{HT}(n=14)$ & $660 \pm 148$ & $308 \pm 59$ & $352 \pm 92$ & $1081 \pm 141$ & $49 \pm 3$ \\
\hline $\mathrm{LT}(n=9)$ & $\begin{array}{l}1550 \pm 40 \\
* \text { vs HT group }\end{array}$ & $\begin{array}{l}1217 \pm 212 \\
* \text { vs HT group }\end{array}$ & $332 \pm 43$ & $706 \pm 175$ & $\begin{array}{l}76 \pm 4 \\
* \text { vs HT group }\end{array}$ \\
\hline
\end{tabular}

${ }^{*} p<0.001$, ANOVA, followed by Scheffe $F$ test; confirmed by Mann-Whitney $U$ test.

standard procedures (Cordeiro et al., 1989; Archibald et al., 1995). There were 1550 and 1114 myelinated axons in the CB and QB respectively; with 2861 and 4832 myelinated axons at the LT and HT sites, respectively. These numbers agree with previous studies (Brushart, 1988). Thus, of the 4832 myelinated axons at the HT, 1114 of them go to the QB, and the expected "probability" of entering a Schwann cell tube to the QB at the parent femoral nerve transection site is $23 \%$ (1114/4832).

A one-sample $Z$ test can compare statistically the observed percentage of "correct" regeneration with that expected by "chance," where the expected SD is estimated from the observed SD (Zar, 1984). The difference between the expected (23\%) and the observed $(49 \%)$ percentages of sensory afferents returning to the QB in the HT group was highly significant $(p<0.0001)$. Obviously, if this same analysis was applied to the motor neuron data in the present study, it would highlight the nonrandom nature of their regeneration accuracy.

To assess the influence of axonal branching on our statistical analysis, we determined the number of myelinated axons proximal and distal to the transection site in the HT group. These numbers give an estimate of the number of myelinated axons that survived at the 7 week time point and the "branching index" present at that time point. As expected, we found some dropout of myelinated axons, at least partially because of the loss of DRG neurons. The HT nerve sites showed an average of $3085 \pm 394$, and $3900 \pm 326$ for the proximal and distal stumps, respectively, compared with the normal of 4832. Thus, the "branching index" is 3900/3085= 1.26. Even when this branching index is taken into account, the difference in regeneration accuracy from the expected chance level still is highly significant $(p<0.0005)$.

\section{The mechanism and possible consequences of specificity generation}

These experiments emphasize the interplay of mechanical (surgical axon alignment) and intrinsic (tropic, trophic, contact recognition) factors in the control of regeneration specificity. Significantly more specificity was generated in sensory and motor systems by distal (LT) compared with proximal (HT) repair, an observation readily explained by proximo-distal changes in the internal topography of the femoral nerve (Brushart, 1988). Similar mechanical control of regeneration specificity has been observed in other model systems (Brushart et al., 1981; Brushart, 1993).

The present study shows a statistically significant tendency for quadriceps sensory afferents to traverse an unstructured gap and reinnervate Schwann cell tubes leading to the quadriceps muscle at much higher than chance levels. That being said, there also is a wealth of previous work that shows that sensory afferent regeneration often is nonspecific. Topographic specificity of vibrissal innervation is lost after repair of the trigeminal nerve (Renehan and Munger, 1986; Arvidsson and Johansson, 1988), and the topography of peroneal nerve projections to the dorsal horn is grossly distorted after regeneration of the rat sciatic nerve across an $8 \mathrm{~mm}$ gap (Brushart, 1990b). In studies comparing the central and peripheral properties of regenerated cat tibial nerve afferents, reinnervation of end organs by modality-inappropriate axons was found to occur $\sim 50 \%$ of the time (Koerber et al., 1989). Physiological studies also have shown that cat muscle spindles normally innervated by group Ia fibers are reinnervated by both $\mathrm{Ia}$ and $\mathrm{Ib}$ fibers after gastrocnemius nerve repair (Collins et al., 1986). Results of such studies often are cited in support of the lack of specificity exhibited by regenerating sensory afferents.

However, these same authors have more recently demonstrated that if one takes into account the degree of accuracy expected by chance (using arguments similar to that discussed above), then the reinnervation of muscle spindles is consistent with some degree of specificity during the reinnervation process (Munson et al., 1988). They suggest that this process is not completely accurate, because afferents directed to the wrong receptor may not be able to correct that error, and once in the wrong location, afferents will innervate a foreign receptor in preference to no receptor. Our anatomical data suggest a limited degree of regeneration specificity for sensory afferents and, in fact, may be the anatomical counterpart to the previous physiological observations. Using a combination of the sequential and simultaneous double-labeling techniques, it now should be possible to determine whether the outgrowth of sensory afferents to the QB is initially random and then undergoes selective pruning similar to that which has been demonstrated previously for the motor neuron projection.

Finally, it is interesting to note the significant correlation between motor- and sensory neuron-regeneration accuracy. As discussed above, it is unlikely that mechanical guidance factors explain the correlation seen in the HT group. Regenerating axons at this level should have equal access to Schwann cell tubes that lead to muscle $(\mathrm{QB})$ or skin $(\mathrm{CB})$. Whatever the exact nature of
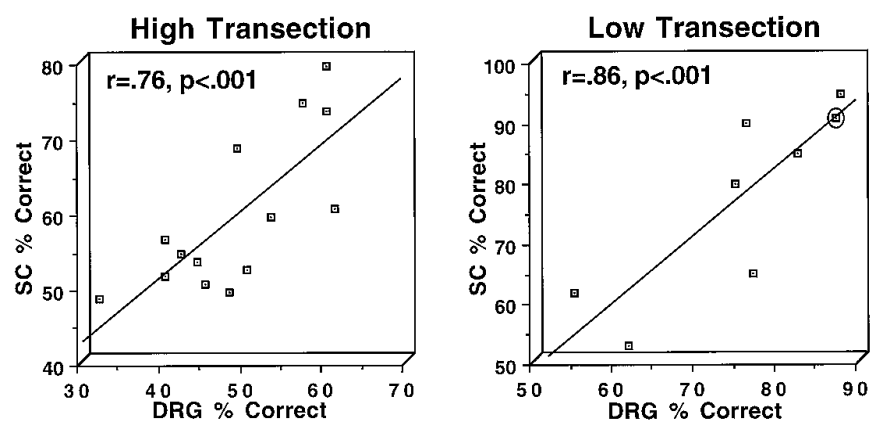

Figure 3. Correlation between percent correct sensory and motor neurons. There was a significant correlation between the percent correct regeneration for motor and sensory neurons returning to the $\mathrm{QB}$. The correlation analysis was performed for each group independently and showed a significant $R$ value within each group. The scatter plot of data with a simple line fit is shown for each repair group. The circled data point in the upper right corner of the LT graph represents two independent superimposed data points. 
such guidance factors, the highly significant correlation $(p<$ 0.001 ) between regeneration accuracy of motor and sensory afferent neurons at this HT level suggests that both neuron classes respond in a parallel manner to such factors. It will be important to determine the molecular basis of preferential reinnervation of motor pathways and to ascertain whether regenerating axons from motor and sensory afferent neurons respond to the same or different molecular cues.

\section{REFERENCES}

Archibald SJ, Shefner J, Krarup C, Madison RD (1995) Monkey median nerve repaired by nerve graft or collagen nerve guide tube. J Neurosci 15:4109-4123.

Arvidsson J, Johansson K (1988) Changes in the central projection pattern of vibrissae innervating primary sensory neurons after peripheral nerve injury in the rat. Neurosci Lett 84:120-124.

Arvidsson J, Ygge J, Grant G (1986) Cell loss in lumbar dorsal root ganglia and transganglionic degeneration after sciatic nerve resection in the rat. Brain Res 373:15-21.

Brown MC, Hardman V (1987) A reassessment of the accuracy of reinnervation by motor neurons following crushing or freezing of the sciatic or lumbar spinal nerves of rats. Brain 110:695-705.

Brown MC, Hopkins WG (1981) Role of degenerating axon pathways in regeneration of mouse soleus motor axons. J Physiol (Lond) 318:365-373.

Brushart TME (1988) Preferential reinnervation of motor nerves by regenerating motor axons. J Neurosci 8:1026-1031.

Brushart TME (1990a) Preferential motor reinnervation: a sequential double-labeling study. Restor Neurol Neurosci 1:281-287.

Brushart TME (1990b) Topographic specificity of peripheral axon regeneration across enclosed gaps. Soc Neurosci Abstr 16:806.

Brushart TME (1993) Motor axons preferentially reinnervate motor pathways. J Neurosci 13:2730-2738.

Brushart TME (1994) Peripheral nerve regeneration: strategies to augment specificity. In: Advances in operative orthopaedics (Stauffer R, ed), pp 205-225. St. Louis: Mosby.

Brushart TME (1996) The effects of pathway and neuronal manipulations on preferential motor reinnervation, in press.

Brushart TME, Henry EW, Mesulam M-M (1981) Reorganization of muscle afferent projections accompanies peripheral nerve regeneration. Neuroscience 6:2053-2061.

Collins WF, Mendell LM, Munson JB (1986) On the specificity of sensory reinnervation of cat skeletal muscle. J Physiol (Lond) 375:587-609.

Cooney WP (1991) Median nerve repairs: the results of treatment. In: Operative nerve repair and reconstruction (Gelberman RH, ed), pp 379-391. Philadelphia: Lippincott.

Cordeiro PG, Seckel BR, Lipton SA, D'Amore PA, Wagner J, Madison R (1989) Acidic fibroblast growth factor enhances peripheral nerve regeneration in vivo. Plast Reconstr Surg 83:1013-1019.

Dodd J, Solter D, Jessel TM (1984) Monoclonal antibodies against carbohydrate differentiation antigens identify subsets of primary sensory neurons. Nature 311:469-472.

Duffy MT, Simpson SB, Liebich DA, Davis BM (1990) Origin of spinal cord axons in the lizard regenerated tail: supernormal projections from local spinal neurons. J Comp Neurol 293:208-222.

Fawcett JW, Keynes RJ (1990) Peripheral nerve regeneration. Annu Rev Neurosci 13:43-60.

Fields RD, Le Beau JM, Longo FM, Ellisman MH (1989) Nerve regeneration through artificial tubular implants. Prog Neurobiol 33:87-134.

Fritzsch B, Sonntag R (1991) Sequential double labelling with different fluorescent dyes coupled to dextran amines as a tool to estimate the accuracy of tracer application and of regeneration. J Neurosci Methods 39:9-17.

Fritzsch B, Wilm C (1990) Dextran amines in neuronal tracing. Trends Neurosci 13:14.

Godement P, Vanselow J, Thanos S, Bonhoeffer F (1987) A study in developing visual systems with a new method of staining neurones and their processes in fixed tissue. Development 101:697-713.

Hall SM (1989) Regeneration in the peripheral nervous system. Neuropathol Appl Neurobiol 15:513-529.

Harsh C, Archibald SJ, Madison RD (1991) Double labeling of saphenous nerve neuron pools: a model for determining the accuracy of axon regeneration at the single neuron level. J Neurosci Methods 39:123-129.

Himes BT, Tessler A (1989) Death of some dorsal root ganglion neurons and plasticity of others following sciatic nerve section in adult and neonatal rats. J Comp Neurol 284:215-230.

Holmes W, Young JZ (1942) Nerve regeneration after immediate and delayed suture. J Anat 77:63-96.

Ide C (1983) Schwann cell basal lamina and nerve regeneration. Brain Res 288:61-75.

Illert M, Fritz N, Aschoff A, Hollander H (1982) Fluorescent compounds as retrograde tracers compared with horseradish peroxidase (HRP). II. A parametric study in the peripheral motor system of the cat. J Neurosci Methods 6:199-218.

Koerber HR, Seymour AW, Mendell LM (1989) Mismatches between peripheral receptor type and central projections after peripheral nerve regeneration. Neurosci Lett 99:67-72.

Konigsmark BE (1970) Methods for the counting of neurons. In: Contemporary research methods in neuroanatomy (Nauta WJH, Ebbesson SOE, eds), pp 315-340. New York: Springer.

Lee MT, Farel PB (1988) Guidance of regenerating motor axons in larval and juvenile bullfrogs. J Neurosci 8:2430-2437.

Madison RD, Archibald SJ (1992) Single neuron level analysis of the accuracy of regeneration of a motor neuron pool to appropriate target muscle. In: Third Vienna muscle symposium (Frelinger G, Deutinger M, eds), pp 80-84. Vienna: Blackwell-MZV.

Madison RD, Archibald SJ, Krarup C (1992) Peripheral nerve injury. In: Wound healing: biochemical and clinical aspects (Cohen IK, Diegelman F, Lindblad WJ, eds), pp 450-480. Philadelphia: Saunders.

Munson JB, Collins WF, Mendell LM (1988) Reinnervation of muscle spindles by groups Ia and Ib fibers is consistent with specificity in the reinnervation process. In: The current status of peripheral nerve regeneration, pp 259-268. New York: Liss.

Raivich G, Kreutzberg GW (1993) Peripheral nerve regeneration: role of growth factors and their receptors. Int J Dev Neurosci 11:311-324.

Ramon y Cajal S (1928) Degeneration and regeneration of the nervous system. New York: Oxford UP.

Renehan WE, Munger BL (1986) Degeneration and regeneration of peripheral nerve in the rat trigeminal system. II. Response to nerve lesions. J Comp Neurol 249:429-459.

Scherer SS, Easter Jr SS (1984) Degenerative and regenerative changes in the trochlear nerve of goldfish. J Neurocytol 13:519-565.

Swett JE, Wikholm RP, Blanks RHI, Swett AL, Conley LC (1986) Motor neurons of the rat sciatic nerve. Exp Neurol 93:227-252.

Weiss P, Edds MV (1945) Sensory-motor nerve crosses in the rat. J Neurophysiol 30:173-193.

Wigston DJ, Kennedy PR (1987) Selective reinnervation of transplanted muscles by their original motoneurons in the axolotl. J Neurosci 7:1857-1865.

Zar JH (1984) The binomial distribution. In: Biostatistical analysis, Vol II, pp 369-405. Englewood Cliffs, NJ: Prentice Hall. 